\title{
The effect of changes in curve geometry on magnitude estimates of road-like perspective curvature
}

\author{
B. N. FILDES and T. J. TRIGGS \\ Monash University, Clayton, Victoria, Australia
}

\begin{abstract}
This study investigated the perception of curvature of perspectively viewed road-like curves in two-dimensional display. The effects of the geometric variables curve radius and curve angle, as well as the influence of experimental instructions, were assessed in a factorial laboratory experiment. Subjects estimated the magnitude of curvature of a series of stimulus curves in relation to a standard view. The results showed that decreasing the curve angle led to perceptual flattening of these curves. Reductions in curve radius resulted in a paradoxical decrease in perceived curvature. Judgments of curvature were the same for uninstructed subjects and those instructed in the meaning of physical curvature. These findings are in contrast to those expected from curve geometry and highlight an illusion in perceived curvature potentially harmful for curve negotiation on the road.
\end{abstract}

In geometric terms, the amount of curvature of an arc of a circle is determined by the radius ( $R$ ); there is an inverse relationship between curvature and radius such that the amount of bending of an arc will increase as the radius decreases. Alternatively, curvature can also be described (as pointed out by ten Brummelaar, 1968) as the rate of change of the arc angle $(\theta)$ with respect to the arc length $(\ell)$, that is, curvature of an arc can be expressed in terms of its angle and arc length. Thus, the physical description of curvature can be in terms of either $\theta$ and $\ell$ or $R$, as shown in the formula:

$$
\text { curvature }=\mathrm{d} \theta / \mathrm{d} \boldsymbol{l}=1 / \mathbf{R} \text {. }
$$

Figure 1 shows the relationship between curvature and the three geometric variables for a simple arc of a circle in plan-view, and the consequences of changes in curvature on $R$ and $\theta$.

It should be noted that changing the radius of an arc will always cause a change in geometric curvature, whereas changes in the arc angle will result in a change in curvature only in conjunction with manipulating the arc length. In other words, curvature is directly related to radius but only indirectly related to arc angle. Figure 2 shows how the curvature of a road is geometrically in-

A version of this paper was presented at the 20th Annual Conference of the Ergonomics Society of Australia and New Zealand, Adelaide, South Australia, in December 1983. This work is part of the Monash University PhD dissertation of the first author (in press). We would like to thank Peter Gordon, Wal Harris, and R. H. Day of Monash University for their valuable assistance with this project, and to Martha Teghtsoonian and other, anonymous reviewers whose comments greatly improved the manuscript. Requests for reprints should be sent to Tom Triggs, Department of Psychology, Monash University, Clayton, Victoria, 3168, Australia.

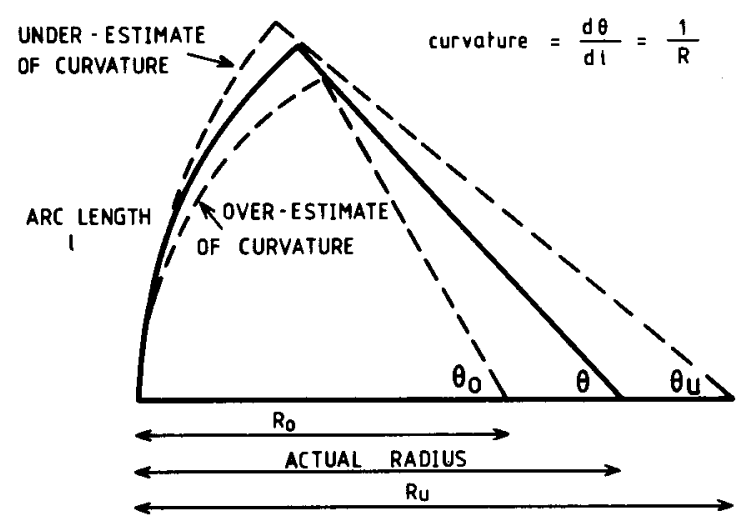

Figure 1. Relationship between curve angle, radius, and length of an arc of a circle in plan-view. Note that for a constant arc length $(l)$, an underestimate of curvature represents an underestimate of curve angle $\left(\theta_{\mu}<\theta\right)$ but an overestimate of curve Radius $\left(\mathbf{R}_{\mu}>\mathbf{R}\right)$.

dependent of the curve angle alone for two road curves of different radius but with the same curve angle.

\section{Subjective Curvature}

Much of the psychological literature dealing with the perception of arcs of circles in plan-view clearly shows that the arc angle can influence subjective curvature. Figures produced by Tolanski (1964), for instance, show a compelling illusion in apparent curvature of a constant radius arc as angle is decreased (see Figure 3.) Virsu (1971a) empirically tested the perceived curvature of simple arcs using a curvature matching technique. He found that subjects underestimated the curvature of small-angle arcs and that their errors greatly increased as the arc angle reduced below $90^{\circ}$. This phenomenon is typically called "perceptual flattening"' (Robinson, 1972). 


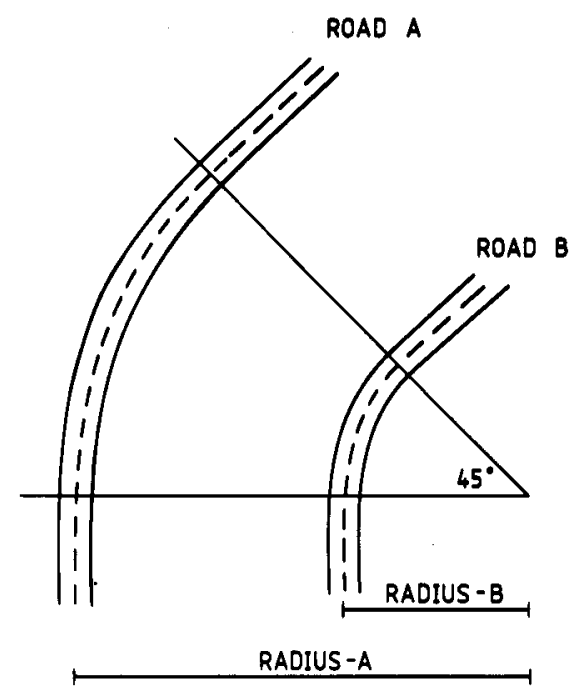

Figure 2. Two road curves viewed from above. Both bends have the same curve angle (they are both $45^{\circ}$ bends), but road $B$ is more curved than road $A$ because it has a smaller radius.

However, with indirect assessment of curvature, for example by asking subjects to judge arc and chord width (Coren \& Festinger, 1967) or the length of the chord (Piaget \& Vurpillot, 1956), it is possible to demonstrate the opposite effect. The nature of the illusion, therefore, is somewhat dependent on how one goes about measuring it.

\section{Theories of Curvature Perception}

There seems to be a general lack of adequate theoretical explanations for the perception of curvature. Virsu (1971b) proposed a rectilinear eye-movement theory of curve perception and argued that perceptual flattening of small arcs could be explained in terms of an inhibition in the feedback mechanism to any nonlinear eye movement. Robinson (1972), however, has questioned the theoretical status of this explanation. Other, more general theories of geometric perception, such as Chiang's (1968) diffraction theory or Blakemore, Carpenter, and George-

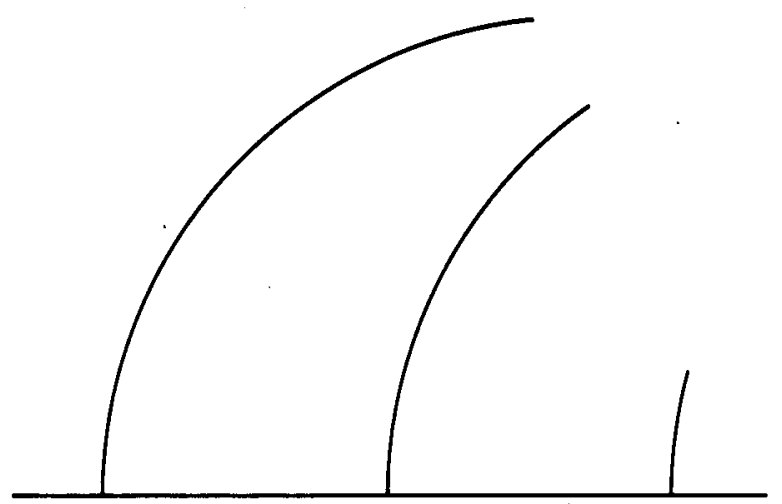

Figure 3. An adaptation of the curvature illusion reported by Tolanski (1964). son's (1970) and Robinson's (1972) angle-expansion theory might eventually prove useful in explaining the perception of perspective curvature. However, before attempting a full theoretical explanation of the phenomenon, we need to understand how the geometric properties of a curve influence the subjective assessment of curvature.

\section{Road Curvature}

In assessing road curvature, it is normally assumed that drivers are sensitive to changes in the curve's radius (Good, 1978; McLean, 1977; Stewart, 1977; ten Brummelaar, 1968, 1975, 1983). Specifications for road delineation, furthermore, stress the need to emphasize the radius aspects of bends in the road by enhancing centerline and edge-line information (Jones, 1961). The role of the deflection angle on curvature is generally considered less important because it is geometrically independent. Yet no studies have confirmed that this independence is reflected in the subjective assessment of curvature on the road.

More recently, highway curves have been designed as transitional or spiral curves consisting of a central section of constant radius and two transitional sections of varying radii which connect the central section with the approach and exit zones. While these curves are thought to enhance the drivers' smooth passage of a curve (Lofthouse, 1978), there is some evidence to suggest that spiral and transitional curves are not perceptually desirable (Stewart, 1977). Hence, the role of radius in the perception of curvature on the road needs further clarification.

\section{The Illusive Curve Phenomenon}

Shinar (1977) argued for an "illusive-curve phenomenon" on the road where drivers underestimate the curvature of an approaching bend as the size of the arc (determined primarily by the curve's angle) or the actual radius of the curve is decreased. Most of the support for the illusive curve came from an experiment in which subjects judged the radius of a series of plan-view simple curves (Shinar, McDowell, \& Rockwell, 1974). Although these results support the classical findings of perceptual flattening of plan-view curves, Shinar's stimuli are not representative of road-curve scenes. Curvature assessment on the road is made on the basis of perspective information, and the outline shape of road bends is geometrically more complex and provides much more curvature information than single-line curves. Thus, the illusive-curve hypothesis really needs to be tested more stringently using controlled road-like curve stimuli before assuming its existence in a driver's assessment of curvature on the road.

\section{Road Curve Assessment}

The curve assessment process has been shown to commence well in advance of the vehicle's entering the curve (Cohen \& Studach, 1977; McLean \& Hoffmann, 1973; Shinar, McDowell, \& Rockwell, 1977). These studies showed that drivers typically scan an approaching bend using many brief $(300-400-\mathrm{msec})$ glimpses scattered 
around the road surface. Hence, the initial curvature assessment process on the road occurs at least $100 \mathrm{~m}$ before the bend, where the rate of change of the perceptual features is relatively low. In other words, a driver's first assessment of a road curve is based on information approximating a static monocular view.

Many researchers have opted to study driving behavior in a laboratory for better control of the variables. In some instances, it has been found that behavior in the laboratory predicts behavior on the road (Evans, 1970, McRuer \& Klein, 1976; Witt \& Hoyos, 1976). Furthermore, Gordon (1966) and McRuer and Klein (1976) have also shown that the majority of curvature information on the road is represented by the edges and centerline of the paved surface. Thus, laboratory stimuli of bends in the road could well be geometric representations of high-contrast edge and centerlines in normal perspective, rather than actual road surfaces or photographs.

\section{Experimental Design}

A magnitude estimation experiment was designed to test the role of curve radius and angle in a driver's estimate of perspective curvature. Two-dimensional representations of three-dimensional curves were adopted as roadlike curve stimuli. From the plan-view curvature results, it was expected that both $R$ and $\theta$ would influence the subject's judgments of curvature.

Magnitude estimation was chosen as the dependent measure for two reasons. First, when curvature estimation of plan-view stimuli was indirect-when the subjects judged something other than the rate of bending of the lines themselves or the curve's radius (Coren \& Festinger, 1967; Piaget \& Vurpillot, 1956)-the results were less systematic. Moreover, the change in effect did not conform with Tolanski's (1964) compelling demonstration of the curvature illusion, and so indirect curvature assessment may well be measuring another phenomenon apart from pure curvature itself. Second, from some earlier results (Fildes \& Triggs, 1982, 1983), it was apparent that, when curve measurement was made in the same mode as one of the independent variables (a radius measure with a radius manipulation for instance), the effect of this variable predominated in the subject's judgments. Thus, it was difficult to partition the effects of the independent variables from measurement error.

As a pretest, a pilot study was performed comparing results using a written number response between the limits of 0 and 100 with 50 as a standard modulus value with a slash across a visual analogue line $100 \mathrm{~mm}$ long. The results showed that both measures gave comparable patterns of responses. Moreover, the subjects preferred a standard view and comparative judgments of the stimuli to an absolute curvature judgment (they felt the latter to be too difficult to make in this context). A comparative assessment also allows better experimental control over the judgments here.

Furthermore, the pilot study highlighted some confusion over the meaning of curvature in this task. Although instructed in physical curvature, subjects were still unsure of whether to respond with an assessment of the rate of bending of the lines representing the road surface or of the absolute amount of bending. Although this confusion might reflect on the way subjects normally perceive curvature, it could also indicate that they might not have fully understood what was required of them in this task. Edwards (1961) highlighted the problem of interpreting data following instructions that were not highly specific. Clearly, there was a need to examine the role of the experimental instructions here.

\section{THE EXPERIMENT}

A factorial, mixed-design experiment was carried out to study the effects of the independent variables of curve radius, curve angle, and experimental instructions on subjects' magnitude estimates of curvature. Testing was carried out with the instruction manipulation as a betweengroup condition, with different subjects in each group. There were two levels of instruction-one providing very detailed information about the meaning of geometric curvature, and the other, no guidance on curvature at all.

\section{Method}

Stimulus materials. The perspective road curve stimuli were all computer-generated line drawings of three single radius arcs representing the edges and the centerline of a road, as well as a line depicting the horizon. The perspective viewpoint was the normal viewing position of a driver in an automobile positioned in the left-hand lane of a two-lane rural highway with a $1-\mathrm{m}$ eye height [National Association of Australian State Road Authorities (NAASRA), 1970]. While vehicles in Australia drive on the lefthand side of the road, the specifications are similar (albeit mirrorimaged) to those recommended for American roads [American Association of State Highway Officals (AASHO), 1965]. Figure 4 depicts a series of typical stimulus scenes where, in each case, the curve is viewed at the start of the bend and the roadlines conclude immediately at the end of the arc.

A series of 60 stimulus scenes were produced incorporating two replications each of 30 different scenes in which three variables were manipulated. The independent variables in the road views represented by the perspective stimulus drawings were curve angle $\left(8^{\circ}, 15^{\circ}, 30^{\circ}, 45^{\circ}\right.$, and $\left.65^{\circ}\right)$, curve radius $(330,500$, and $670 \mathrm{~m})$, and curve direction (left and right). The variable levels were selected from the values typically used in road design for restricted terrain highways (NAASRA, 1970). Stimulus materials consisted of 35-mm slides of white lines on a black background which were photographed from the A4 drawings produced on a Hewlett-Packard page plotter, Type 7221A.

Procedure. Two groups of 22 subjects each, composed of drivers holding a current Australian driving licence, were recruited and tested using a group presentation procedure. The subjects had not participated in any previous curvature-estimation experiments. One group of subjects was assigned to the definitive-instruction condition and given both task training and comprehensive instruction in the meaning of physical curvature. They were directed not to respond to angle but to attend only to changes in the bending of the lines representing the road surface. Rate of bending was expressed in terms of the curve's radius, the amount of change in direction of the road surface, the perceptual effects, and the steering and speed consequences for curve negotiation, to fully emphasize geometric curvature. They were subsequently tested to ensure that they had understood what was meant by physical curvature. The second group 


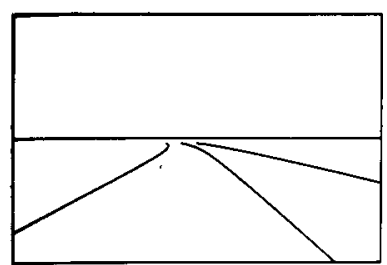

e70m $\times$ 8deg LH

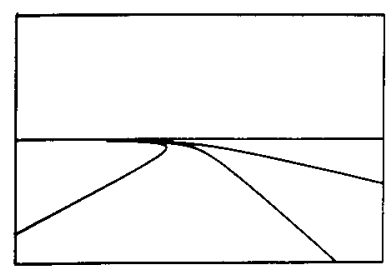

$600 \mathrm{~m} \times 30 \mathrm{deg}$ LH

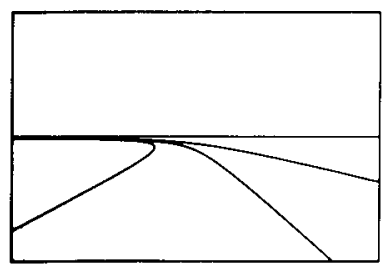

ssom $\times$ esdeg LH

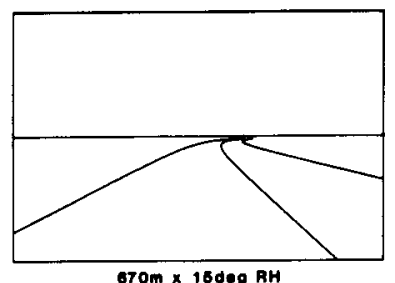

$670 \mathrm{~m} \times 18 \mathrm{deg} \mathrm{RH}$

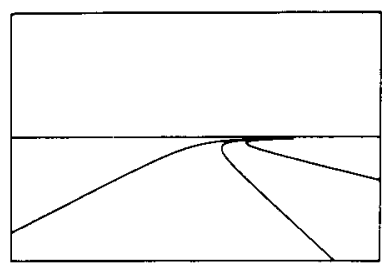

$500 \mathrm{~m} \times 30 \mathrm{deg} \mathrm{RH}$

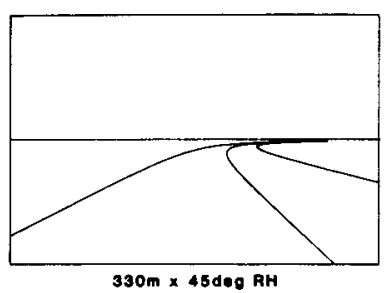

Figure 4. A series of right-hand and left-hand stimulus views presented to the subjects during the experiment.

of subjects received minimal instruction consisting only of task instructions or how to respond to each curve stimulus. These subjects were simply asked to assess the amount of curvature for each test slide without reference to what was meant by geometric curvature.

The experimental task was to estimate the amount of curvature of each test slide in relation to a standard view presented. Subjects in both groups were asked to assign numbers greater than $\mathbf{5 0}$ for more curved stimuli and below 50 for less curved material. No range limits were specified in this experiment for the response scale, and the subjects were encouraged to use whatever range of numbers they thought appropriate for otheir responses. The standard was a curve of $500 \mathrm{~m}$ radius and $65^{\circ}$ angle (which constituted a midrange radius value and a fill screen angle view with all lines concluding at the edge of the projection screen) and was continuously projected adjacent to and left of the stimulus scenes. Both stimulus and standard views were front-projected onto Australux 1,200 $\times 1,200 \mathrm{~mm}$ projection screens, using Kodak Carousel $S$ projectors.

Presentation order of the stimuli was randomized for the angle and radius variables, but blocked into left-hand and right-hand sequences for ease of presentation and to reduce the task demands on the subjects. Presentation time for each test stimulus was $15 \mathrm{sec}$, and the subjects' curvature estimates were recorded on separate labeled pages of a response booklet. Seating was evenly distributed around the projection screens, and the subjects were discouraged from communicating during the experiment.

Statistical analysis. Estimates of perceived curvature were recorded and analyzed using analysis of variance and the omegasquared statistic. This latter measure allows one to estimate the strength of statistical association in terms of the percentage of variance accounted for by a particular manipulation (Hays, 1963; Vaughan \& Corballis, 1969). Statistical effects can then be graded in terms of strength of effect on the basis of the omega-squared value, and thus the stronger effects can be selected for more detailed attention (Keppel, 1973).
One-sample $t$ tests between the means and the modulus were used to identify the source of any significant differences. Hays (1970) recommends the use of $t$ tests for post hoc comparison following a significant overall effect. However, McNemar (1969) points out that multiple post hoc testing can capitalize on chance factors and recommends a reduction in the significance level (to, say, $1 \%$ ) to guard against Type I errors. A probability level of $p<.01$ was therefore adopted for all post hoc comparisons here.

\section{Results}

This experiment was primarily concerned with the effect of the variables of curve radius, curve angle, and experimental instruction on the subject's judgments of curvature. Since the view of the curves was not from the center of the road, it was most likely that perceived curvature would differ for right-hand and left-hand curves. Indeed, previous results (Fildes, 1979; Fildes \& Triggs, 1982; Triggs, Harris, \& Fildes, 1979) had highlighted direction assymetries in the assessment of curve direction. Unfortunately, any effect of curve direction in this experiment would be meaningless, since the judgments for left-hand and right-hand curves were made in relation to a direction-dependent modulus. Hence, curve direction was not considered in any subsequent analysis.

Curve angle. The results presented in Figure 5a show that this variable had a significant effect on the subjects' judgments $\left[F(4,168)=100.4, p<.001, \omega^{2}=.386\right]$. The angles of $8^{\circ}$ and $15^{\circ}$ were judged significantly less than $50[\mathrm{t}(21)=12.9$ and 7.2 , respectively, $\mathrm{p}<.001$, two tailed] and the angles of $45^{\circ}$ and $65^{\circ}$ were assessed as significantly greater than the modulus $[\mathrm{t}(21)=3.8$ and 4.6 , $\mathrm{p}<.001$, two-tailed]. This variable produced the strongest effect observed in this experiment, capturing $98 \%$ of all the experimental variance. The form of the psychophysical relationship for angle is nonlinear, but interestingly is not a simple logarithmic or power function.

Curve radius. The factor of curve radius, shown in
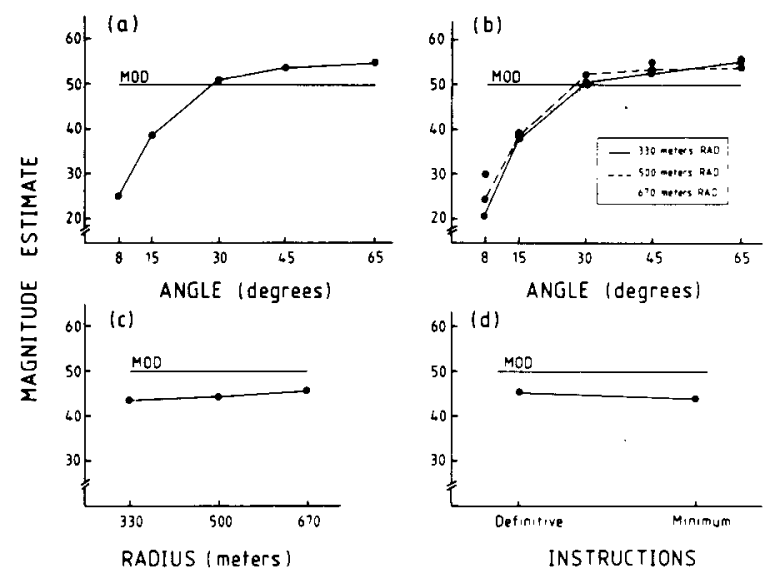

INSTRUCTIONS

Figure 5. Magnitude estimates of curvature for the three variables tested. The main effects of curve angle (1), curve radius (c), and the interaction between angle and radius (b) were all significant at $p<.05$. The instruction variable (d), however, was not significant. Omega-squared rankings supported the significant order listed where angle captured $97.5 \%$ of the experimental variance. 
Figure 5c, was significant $[\mathrm{F}(2,84)=4.8, \mathrm{p}<.01$, $\left.\omega^{2}=.002\right]$, although not in the geometrically expected direction, that is, $330 \mathrm{~m}$ radius was judged the least curved of all the stimuli presented and curvature approached the modulus value as the radius increased. One-sample $t$ tests (two-tailed), reveal significant differences from the modulus for each level of curve radius [at $330 \mathrm{~m}, \mathrm{t}(21)=6.0$, $\mathrm{p}<.001$; at $500 \mathrm{~m}, \mathrm{t}(21)=5.9, \mathrm{p}<.001$; and at $670 \mathrm{~m}$, $\mathrm{t}(21)=4.5, \mathrm{p}<.001]$. Radius as a main effect, however, was only $0.5 \%$ the strength of effect of angle.

Radius $\times$ angle. A significant interaction was observed between curve radius and angle $[F(8,336)=6.6, p<$ $.001, \omega^{2}=.006$ ], as shown in Figure 5b. Small-radius, small-angle bends were judged to be less curved, and estimates became more accurate as both angle and radius increased. A simple main effects analysis was carried out for radius at each level of curve angle, and showed that the source of this interaction was at $8^{\circ}[\mathrm{F}(2,84)=38.5$, $\mathrm{p}<.001$ ]; radius was not significant at any other level of curve angle $(p>.05)$.

Experimental instructions. The type of instructions given in this experiment did not significantly influence the results obtained[F(1,42) $\left.<1.0, p>.05, \omega^{2}=0\right]$, as shown in Figure 5d. Subjects responded much the same regardless of which level of instruction they had received. This factor did not interact with any other variable and essentially showed no statistical association with the dependent variable. Moreover, there was practically no difference in variance between the two instruction conditions (standard deviation $=18.1$ for the definitive group compared with 18.2 for the minimal group).

Arc length. It was noted in the introduction that curvature can be defined geometrically in terms of either radius $(R)$ or angle $(\theta)$ and arc length $(\ell)$. When radius and angle in the stimulus set in this experiment are manipulated, the arc length is also varied by nature of the interrelationships among these three parameters. Since Shinar (1977) and Virsu (1971b) both argued that the arc length directly influences perceived curvature, it seemed appropriate to reanalyze the data in terms of this variable

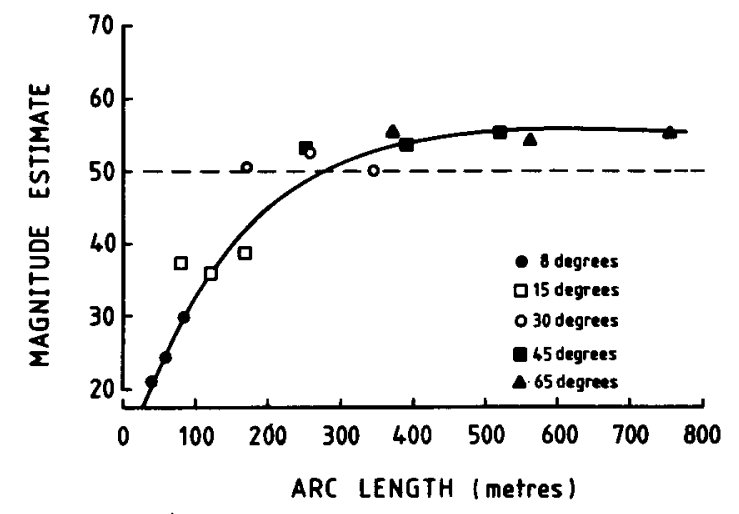

Figure 6. Magnitude estimate results replotted against an arc length continuum. Each curve angle value is highlighted to indicate the importance of this variable. alone. Figure 6 shows the responses as a function of arc length.

A one-way analysis of variance was performed for the arc length variable and, not surprisingly, revealed a significant effect $[F(14,602)=72.8, p<.001]$. However, the form of the relationship between arc length and magnitude estimates was more interesting. Small arc lengths, in general, were judged less curved than large arc lengths, particularly for values below $300 \mathrm{~m}$. Moreover, when the various angle data points are considered, the curvature estimates at each level of curve angle (with the exception of $8^{\circ}$ stimuli) were reasonably constant, even though they actually represented three different levels of physical curvature. This suggests that curvature judgments in this experiment were more sensitive to plan-view curve angle than to arc length per se.

\section{DISCUSSION}

When asked to estimate the amount of curvature of a series of geometric perspective representations of roadcurve stimuli with both the curve radius and angle changing, subjects' judgments were predominantly influenced by the curve's deflection angle. Small-angle bends were seen as less curved than the standard, whereas large-angle bends were seen to be more curved, and this variable accounted for most of the experimental variance, in spite of the fact that angle by itself does not physically influence the amount of curvature of an arc of a circle.

Radius effects, although significant, were of considerably less strength and, interestingly, opposite to that predicted by curvature geometry. In other words, smallradius bends were judged to be less curved, rather than more curved, than the standard view. This result was further accentuated by the significant interaction in the responses between radius and angle. Subjects in this experiment clearly responded to small-radius, small-angle curves as the least curved of all the scenes presented, even though they were, in fact, the most curved.

Several aspects of these results need to be discussed. First, the overwhelming effect of angle that was observed in this experiment was unexpected from either previous research or the properties of geometric curvature. Morover, this effect was not influenced significantly by the specific instructions. One group of subjects had been comprehensively instructed in geometric curvature prior to participating in the experiment (in several alternative forms to ensure as far as possible that they had fully understood the concepts). Additionally, they had also been asked not to respond simply to the amount of the curve that was visible when making their judgments. Nevertheless, they still responded counter to the actual geometry of the curve. Clearly, the subjective interpretation of curvature observed here is noticeably different from that specified by curve geometry.

Secondly, the radius paradox, where the most curved stimuli were judged to be the least curved, has also not been formally stated before. Shinar et al. (1974) reported 
perceptual flattening for their plan-view single-line curves, and, although they also observed an interaction between curve angle and radius similar to the one reported here, they did not comment on the paradoxical nature of these results. Moreover, there was no a priori reason to expect that the plan-view findings of Shinar et al. (1974) would necessarily generalize to perspective curve assessment. The cues available for assessing curvature on the road are quite different from single lines in plan-view. Nevertheless, the similarity in the results between both experiments suggests that estimates of plan-view curvature are not totally unrelated to those of perspective representations of road curves.

Third, the critical variable appears to be curve angle. Shinar (1977), Shinar et al. (1974), and Virsu (1971a, 1971b) all suggested that subjects base their estimates of curvature on judgments of arc length. Indeed, this notion has credence with road designers in that a minimum sight distance is also specified in the geometric design of rural road curves (AASHO, 1965; NAASRA, 1970). The results from this experiment, however, do not support such a contention. Although curvature judgments generally increased in accuracy as the arc length increased, the results suggest that angle, primarily, and radius, to a lesser extent, were more closely involved in the assessment of curvature than arc length per se. The relationship observed between arc length and curvature in this experiment, therefore, would have been expected given that perceptual flattening occurred when both angle and radius reduced.

The fourth point involves the possibility of response bias in the data. According to the modulus of magnitude estimation, the mean value for the $65^{\circ} 500-\mathrm{m}$ stimuli in Figure $5 \mathrm{~b}$ was expected to be 50 . Subjects' judgments for these values, however, were greater than the modulus, suggesting that the task influenced the large-angle results. Furthermore, the response function for angle in this experiment was not a traditional logarithmic or power relationship. Poulton $(1968,1969)$ argues that a traditional psychophysical function is obtained in these experiments only when the standard is midpoint in the range of values manipulated. While the standard radius value conformed with Poulton's "model," the modulus value for angle was always at the extreme end of the stimulus angle range. Hence, anomalies observed in the responses for the smallangle stimuli may approximate Stevens's (1966) recruitment phenomenon.

Relaxing the range limit constraint imposed in the pilot experiment appears to have had little influence on the responses, since there were no instances of curvature estimates recorded beyond the upper limit of 100 . The subjects seem to have adopted this value as an upper limit, even though they had had no prior experience with the task and were actively encouraged to use the range of numbers with which they felt most comfortable. Furthermore, as there was very little skewness in the frequency distributions of the responses, it would appear that the magnitude estimates were not influenced significantly by the scale.

The final comment is in relation to the consequences of these findings for road users. The results obtained in this experiment indicate that when drivers are asked to make curvature judgments under conditions of varying curve angle and radius (a situation commonly experienced as a driver moves along a curved two-lane highway), they will primarily make that judgment on the basis of the curve's deflection angle. The radius of curvature (which is the more relevent geometric variable) is likely to be misinterpreted by drivers in their assessment, particularly for curves of small angles. As current design standards for roads assume that drivers are primarily sensitive to curve radius, these results highlight a potentially dangerous illusion in the perception of curvature during the negotiation of bends in the road.

\section{REFERENCES}

American Association of State Highway Officials (1965). A policy on design of rural highways. Washington, DC: Author.

Blakemore, C., Carpenter, R. H. S., \& Georgeson, M. A. (1970). Lateral inhibition between orientation detectors in the human visual system. Nature, 228 37-39.

ChIANG, C. (1968). A new theory to explain geometrical illusions produced by crossing lines. Perception \& Psychophysics, 3, 174-176.

Cohen, A. S., \& Studach, H. (1977). Eye movements while driving cars around corners. Perceptual and Motor Skills, 44, 683-689.

COREN, S., \& Festinger, L. (1967). An alternative view of the "Gibson normalization effect." Perception \& Psychophysics, 2, 621-626.

EDWARDs, W. (1961). Costs and payoffs are instructions. Psychological Review, 68, 275-284.

Evans, L. (1970). Automobile-speed estimation using movie film simulation. Ergonomics, 13, 231-237.

FiLDEs, B. N. (1979). Delineation of rural roads at night: The effect of visual blurring and luminance changes on estimating curve direction. Unpublished Honours Thesis, Monash University.

Fildes, B. N., \& Triggs, T. J. (1982). The effects of road curve geometry and approach distance on judgments of curve exit angle. Procedings of the Eleventh Australian Road Research Board Conference, 11, 135-144.

Fildes, B. N. \& Triggs, T. J. (1983). The role of curve radius and angle in the subjective assessment of road curvature. Proceedings of the 20th Ergonomics Society Conference, 20, 73-86.

Good, M. C. (1978). Road curve geometry and driver behaviour (Special Report No. 15). Melbourne: Australian Road Research Board.

GoRdon, D. A. (1966). Experimental isolation of driver's visual input. Public Roads, 33, 266-273.

HaYs. W. L. (1963). Statistics for psychologists. New York: Holt, Rinehart and Winston.

Hays, W. L. (1970). Statistics. London: Holt, Rinehart and Winston. JoHNSTON, I. R. (1982). Modifying driver behaviour on rural roadsA review of recent research. Proceedings of the Eleventh Australian Road Research Board Conference, 11, 115-124.

JONES. J. H. (1961). The geometric design of modern highways. London: Spon.

KEPPEL, G. (1973). Design and analysis: A researcher's handbook. Englewood Cliffs, NJ: Prentice-Hall

Lofthouse. W. A. (1978). Trasitional curves on bends. The Highway Engineer, 25, 2-4.

MCLEAN, J. R. (1977). The inter-relationship between accidents and road alignment (Internal Report. AIR 000-68). Melbourne: Australian Road Research Board.

McLean, J. R., \& HoffmanN. E. R. (1973). The effects of restricted 
preview on driver steering control and performance. Human Factors, $15,421-430$.

McNemar, Q. (1969). Psychological statistics (4th ed.). New York: Wiley.

MCRUER, D. T., \& KLEIN, R. H. (1976). Comparison of human driver dynamics in an automobile on the road with those in simulators having complex and simple visual displays. Paper presented at the 55th Annual Meeting of the Transportation Research Board, Washington, DC.

National association of Australian State Road Authorities (1970). Policy for geometric design of rural roads (4th ed.). Sydney, NSW, Australia: Department of Main Roads.

Piaget, J., \& Vurpillot, E. (1956). La surestimation de la courbure des arcs de cercle. Archives de Psychologie, 35, 215-232.

Poulton, E. C. (1968). The new psychophysics: Six models for magnitude estimation. Psychological Bulletin, 69, 1-19.

Poulton, E. C. (1979). Models for biases in judging sensory magnitude. Psychological Bulletin, 86, 777-803.

RoBinson, J. O. (1972). The psychology of visual illusion. London: Hutchinson University Library.

SHINAR, D. (1977). Curve perception and accidents on curves: An illusive curve phenomenon? Zeitschrift für Verkehrssicherheit, 23, 16-21.

ShINAR, D., MCDOWELl; E. D. \& RockWELL, T. H. (1974). Improving driver performance on curves in rural highways through perceptual changes (Interim Report, Project EES 428). Columbus, Ohio: Ohio State Unviversity, Systems Research Group.

SHINAR, D., McDowell, E. D. \& Rockwell, T. H. (1977). Eye movements in curve negotiation. Human Factors, 19, 63-71.

STEvens, S. S. (1966). Power-group transformations under glare, masking and recruitment. Joumal of the Acoustical Society of America, 39, 725-735.
Stewart, D. (1977). The case of the left-hand bend. The Highway Engineer, 24, 12-24.

Ten BrummelaAr, T. (1968). The deflection angle, its derivatives and their relation to curvilinear road alignment. Proceedings of the Fourth Australian Road Research Board Conference, 4, 697-711.

Ten BrummelaAr, T. (1975). Where are the kinks in the alignment? Transportation Research Record, No. 556, 35-50.

TEN BrummelaAR, T. (1983). The reversal point in the perspective road picture. Australian Road Research, 13, 123-127.

TolANSKI, S. (1964). Optical illusions. Oxford: Pergamon.

Triggs, T. J., Harris, W. G., \& Fildes, B. N. (1979). Delineation cues on rural roads at night: A laboratory-based study of curve direction estimation. (Internal Report AIR 266-2). Melbourne: Australian Road Research Board.

Vaughan, G. M. Corbalus, M. C. (1969). Beyond tests of significance: Estimating strength of effects in selected ANOVA designs. Psychological Bulletin, 72, 204-213.

VIRSU, V. (1971a). Tendencies to eye movements and misperception of curvature, direction, and length. Perception \& Psychophysics, 9. 65-72.

VIRSU, V. (1971b), Underestimation of curvature and task dependence in visual perception of form. Perception \& Psychophysics, 9, 339-342.

WITT, H., \& HoYos, C. G. (1976). Advanced information on the road: A simulation study of the effect of road marking. Human Factors, 16, 521-532.

(Manuscript received November 28, 1983; revision accepted for publication January 14, 1985). 